\title{
Greenhouse microclimate environment adaptive control based on a wireless sensor network
}

\author{
Lina Wang, Binrui Wang* \\ (College of Mechanical and Electronic Engineering, China Jiliang University, Hangzhou 310018, China)
}

\begin{abstract}
Environmental parameter measurements from various sensors spatially distributed in greenhouse crops can be used to create an accurate and detailed depiction of the climate in different regions of the greenhouse. Microclimate environments have an impact on crop yield, productivity, quantitative and qualitative characteristics of plants, and various plant diseases. In this study, an automatic monitoring system based on a wireless sensor network was designed to monitor the survival rate of greenhouse crops. The system design includes a sensor model selection and placement process, host computer monitoring software and communication module design. The Zigbee protocol was used for wireless communication between the nodes. The proposed fuzzy-PID controller was easy to design and highly adaptive to the measurement errors of the sensors. The test results show that the system valuable for monitoring the environment in the greenhouse, where it was successful in controlling and maintaining an optimal microclimate condition.
\end{abstract}

Keywords: greenhouse, microclimate control, wireless sensor network, fuzzy-PID control

DOI: $10.25165 /$ j.ijabe.20201303.5027

Citation: Wang L N, Wang B R. Greenhouse microclimate environment adaptive control based on a wireless sensor network. Int J Agric \& Biol Eng, 2020; 13(3): 64-69.

\section{Introduction}

Facility horticulture that involves modern agricultural engineering, machinery, technology and management is used to improve the local environment and allow crops to adapt to growth temperatures, light, water, gas, fertilizer and other microclimate parameters in a controlled environment industry, that contains crop cultivation management technology, environmental control, and greenhouse engineering technology along with supporting equipment ${ }^{[1-3]}$. The greenhouse regulatory mechanism is complex and involves numerous environmental conditions necessary for growing crops, as shown in Figure 1. Controlling the environmental changes in a greenhouse is challenging because of the complex environmental characteristics ${ }^{[4-6]}$. Precision agriculture is crucial to modern greenhouse cultivation practices, which include recording crop conditions, providing crop growth regulation and monitoring crop field variability ${ }^{[7,8]}$.

In recent years, wireless sensor networks (WSNs) have been widely used in greenhouse microclimate environments ${ }^{[9]}$. Traditional monitoring systems are common in greenhouse cultivation, including wired communication between sensing and computing devices ${ }^{[10,11]}$. The WSN automatically gathers information hundreds or thousands from sensor node base stations or sink nodes that are used to acquire parameters under certain conditions and then transfers these parameters. WSNs exhibit features that facilitate placement and ad hoc communication; along with improved sensing abilities. The network has the ability to continuously record dynamic environmental parameter changes in

Received date: 2019-03-12 Accepted date: 2020-04-19

Biographies: Lina Wang, PhD, Lecturer, research interest: intelligent regulation of greenhouse microclimate environment. Email: 19A0102172@ cjlu.edu.cn;

*Corresponding author: Binrui Wang, $\mathrm{PhD}$, Professor, research interest: machine vision and inspection. College of Mechanical and Electronic Engineering, China Jiliang University, Xueyuan Street, 258, Hangzhou 310018, China. Tel: +86-571-86914488, Email: wangbinrui@163.com. various greenhouse environments ${ }^{[12,13]}$. However, sensor networks have limitations and require additional boundary nodes to enable the precise control of climate change in the greenhouse. In addition, factors such as temperature, humidity, light, water, and other microclimate parameters; are important concerns. Adaptive control schemes should be considered for the complex nonlinear greenhouse control system with microclimate parameters ${ }^{[14]}$.

In the last few years, much attention to the intelligent algorithm has been paid. Major efforts have been devoted to the control of greenhouse environments. Thus, diverse intelligent methods, including adaptive, robust, predictive, fuzzy, and nonlinear controls have been proposed for use as a greenhouse microclimate control ${ }^{[15-19]}$. These studies are of great significance for practical engineering applications of greenhouse production. However, most of these methods are theoretically complex, or difficult to carry out in the actual greenhouse. The fuzzy control method for nonlinear systems analysis has been recently developed. A fuzzy controller was proposed to keep the internal greenhouse temperature using hot air when the temperature decreases at night ${ }^{[20]}$. The architecture of proportional, integral, and derivative (PID) controllers is simple enough ${ }^{[21]}$. We can take advantage of the advantages of fuzzy PID methods for use in a greenhouse control system that is a complex strongly coupled nonlinear system $^{[2,22,23]}$

The microclimate system in a greenhouse is an important control problem that is crucial to the proper growth of the crops. A microclimate control can maintain a greenhouse temperature and humidity that is suitable for crop growth. This study investigates the WSNs and host computer monitoring that enable actuators to calculate and control the microclimate system. The sensor models containing temperature, humidity and illumination sensor are presented to allow the control system to monitor key environmental parameters. The host computer monitoring system can automatically collect and transport data, and send real-time data and alarm messages to the manager via a WSN when the parameter exceeds the set threshold. A fuzzy-PID controller that is highly 
adaptable, and easy to design is utilized in this study for adjusting the measurement errors of the sensors. The proposed intelligent system can create and preserve an ideal climate condition in the greenhouse.

The remainder of this paper is as follows: Section 2 describes the system requirements and parameters analysis. Then, the sensor model selection and placement, host computer monitoring system and communication module design are discussed in Section 3. The test results from the greenhouse monitoring system are presented in Section 4. Finally, conclusions are given in Section 5.

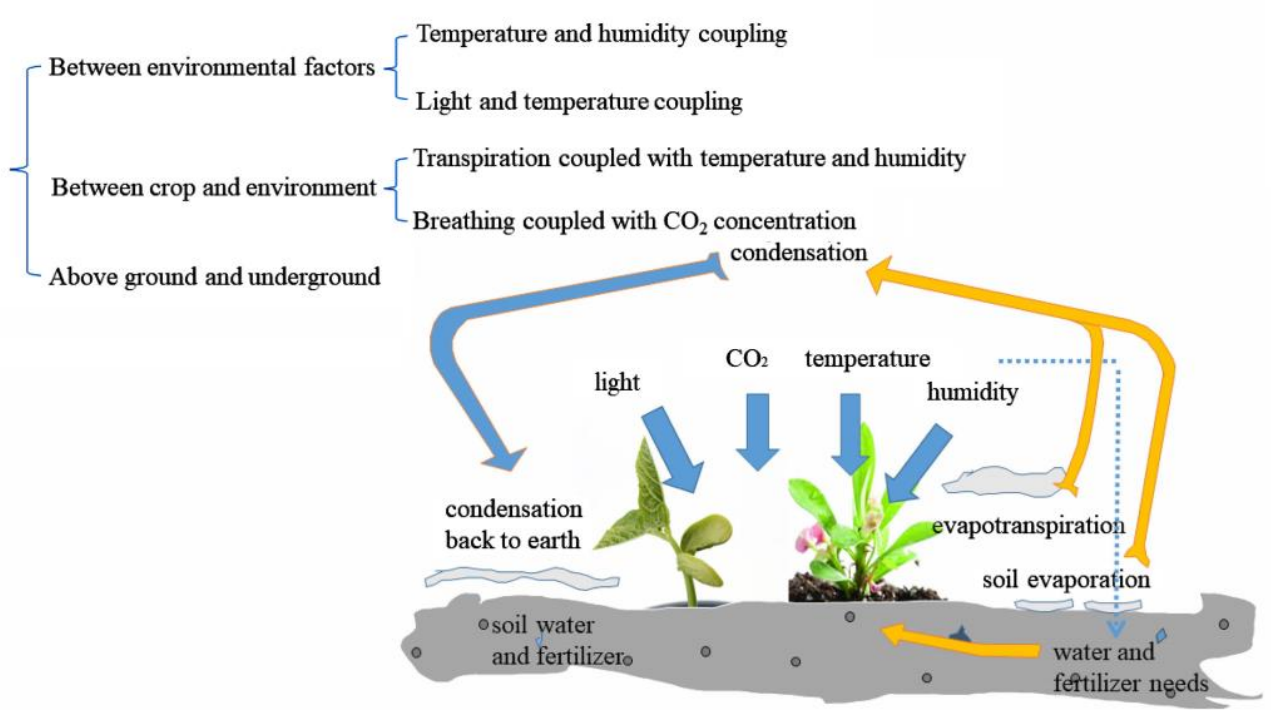

Figure 1 Schematic diagram of coupling relationship of greenhouse microclimate parameters

\section{System requirements and parameter analysis}

Each greenhouse is equipped with a wireless sensor network node that is connected to temperature, humidity and light sensors to measure the internal and external values of the greenhouse. The monitoring system can collect and send the sensor data from all greenhouses, as shown in Figure 2. If the threshold for a parameter is exceeded, the alarm can be issued. The base station with host computer monitoring software is located in a place where farmers can easily access real-time monitoring data[ ${ }^{24]}$. The intelligent control algorithm can calculate the deviation between the predicted value and the actual value, thereby adjusting the sensor equipment to provide suitable climate growing climatic conditions for the greenhouse crop.

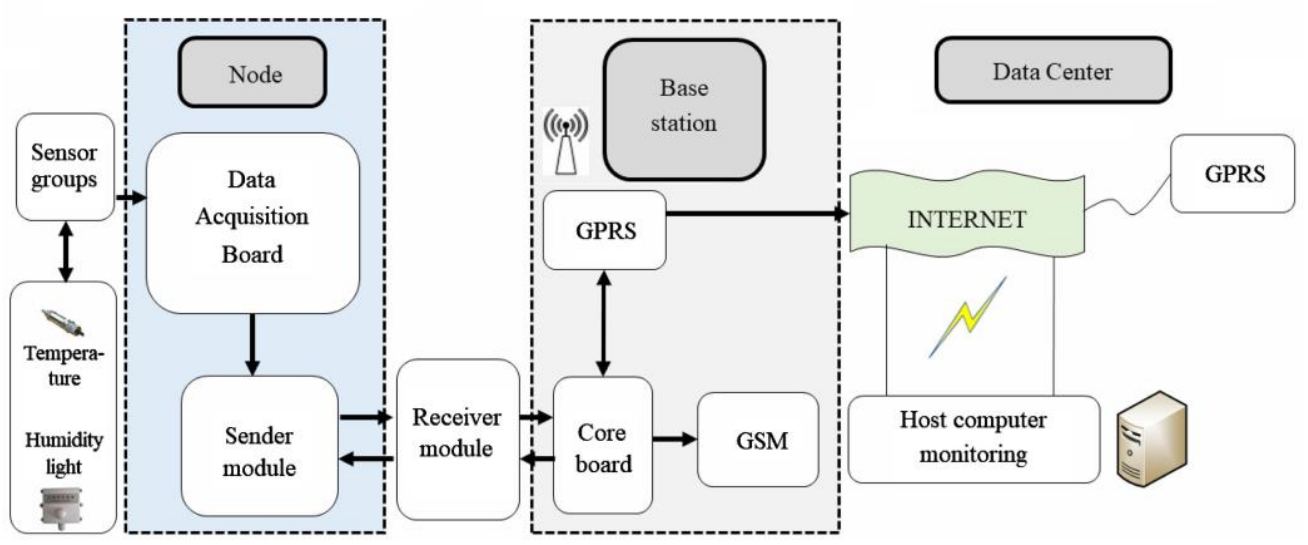

Figure 2 Monitoring system of greenhouse based on WSNs

Temperature is a key parameter that influences the crop growth and the physiological processes of plants within a certain range [ ${ }^{8,25]}$. When the temperature is below the required temperature range, the normal physiological activities of crops will become decrease, which will affect the normal growth of crops. When the temperature is higher in the crops than the required temperature range, crop respiration will be affected by interference, and crop transpiration can also be delayed, thus inhibiting the growth of the crops. Therefore, it is important to monitor the temperature parameters in the greenhouse.

If the air humidity is too high, the transpiration of the crop will be weakened, and the ability to transport minerals and nutrients will also decrease. If the long-term humidity is too low because there is moisture inside the blade, the external water pressure is low, the water pressure differential will be too high, which will cause necrosis in the leaf seriously affect the growth of the crop ${ }^{[22,26,27]}$. Therefore, it is very important to control the humidity within a suitable range by monitoring the humidity of the air in the greenhouse in real-time.

If the light intensity is too high, water loss will occur in the cells of the crop. When the light intensity is too low, the photosynthesis efficiency will decrease. Furthermore, when the light intensity falls below to a certain range, the photosynthesis carried out by the crop will not be enough for the respiratory action to take place effectively, and the crop will stop growing. Therefore, the light intensity is also very important for crops. In addition, these environmental parameters are interactive. The change in temperature affects the humidity. The higher the temperature is, the greater the loss of moisture into the air which leads to a corresponding increase in humidity. The light intensity 
will change the temperature ${ }^{[4]}$. It can be seen that the growth and development of crops are affected by the interaction of these parameters.

\section{System hardware and software design}

\subsection{Sensor model selection and placement}

As shown in [9], the reliability of temperature and relative humidity measurements is significantly affected by the solar radiation intensity in the real environment. The WSN selection is very important in controlling greenhouse microclimate conditions. When the sensor collects information and sends data to the host computer, monitoring system, the station can make decisions and drive actuators. In this work, we selected some temperature, humidity, and illumination sensor styles.

\subsubsection{Temperature sensor DS18B20}

The DS18B20 is a commonly used digital temperature sensor, which outputs digital signals, has the characteristics of small size, low hardware overhead, strong anti-interference ability, and high accuracy. It can measure temperatures from $-55^{\circ} \mathrm{C}$ to $125^{\circ} \mathrm{C}$, with a precision of $\pm 0.5^{\circ} \mathrm{C}$. The sensor can be controlled directly by a single chip microcomputer without the need for A/D conversion. The circuit diagram is shown in Figure 3. The DQ terminal is the signal end, and this design is connected with P0.6 microcontroller, the GND terminal is directly grounded, and the $\mathrm{VCC}$ is connected to a $3.3 \mathrm{~V}$ power source.

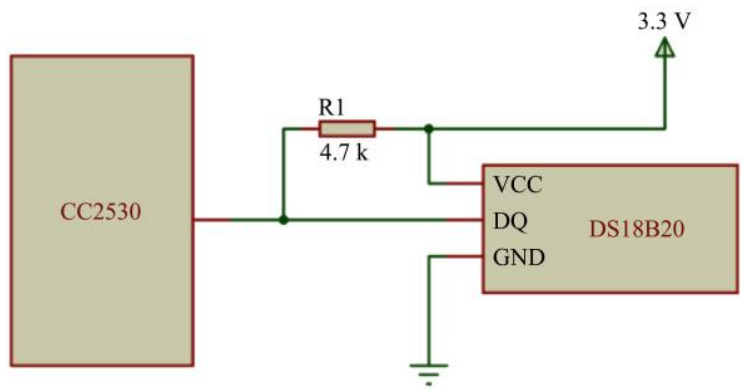

Figure 3 DS18B20 temperature sensor circuit

\subsubsection{Humidity sensor DHT11}

The DHT11 is a single-wire serial interface and the output is a digital signal. The sensor includes a resistive humidity sensing element and an NTC temperature measuring element and is connected to a high-performance 8-bit microcontroller. Therefore, this product has the advantages of excellent quality, ultra-fast response, strong anti-interference ability, and high-cost performance. Because the DHT11 can also measure temperature, compared with the DS18B20, its temperature measurement accuracy is far less it is only $\pm 2{ }^{\circ} \mathrm{C}$, therefore, we only used it to measure air humidity. The circuit diagram is shown in Figure 4 DATA is connected to the microcontroller P0.7 port.

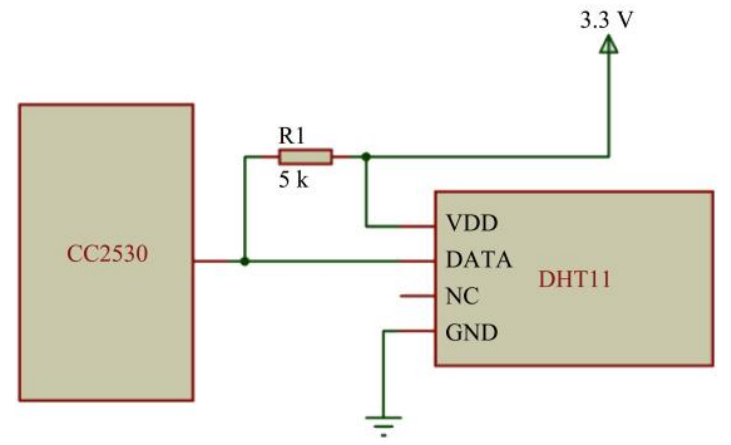

Figure 4 DHT11 humidity sensor circuit

\subsubsection{Intensity of illumination sensor GY-302}

The solution for light intensity monitoring using the BH1750FVI is digital. It is based on the IIC interface and can collect light intensity. Its power supply 3-5 V, data range 0-6535, sensor built-in 16-bit $\mathrm{AD}$ converter, direct digital output, omit complex calculations and omit calibration. Spectral characteristics close to visual acuity allow high-precision measurement of 1 lux over a wide range of brightness. The Gy-302 is a sensor that uses the BH1750FVI chip. The circuit diagram is shown in Figure 5. SCL is the clock pin of the IIC bus, SDA is the data pin of the IIC bus, and ADDR is the address pin of the IIC device, which is directly grounded. SCL is connected to a P1.4 microcontroller, and SDA is connected to a P1.5 microcontroller.

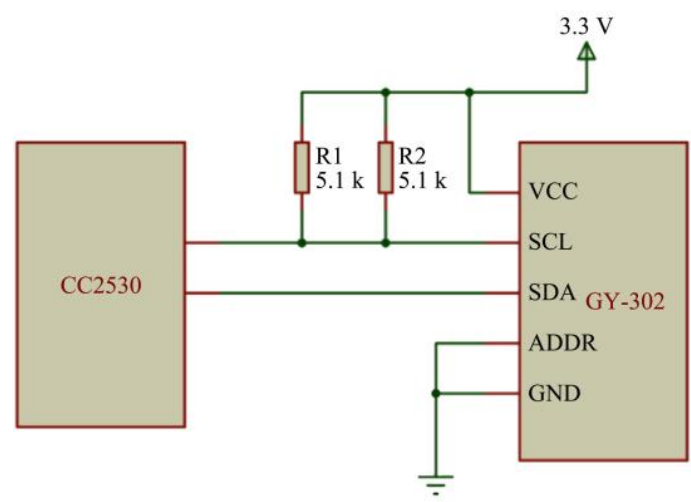

Figure 5 GY-302 light sensor circuit

3.1.4 Data acquisition program design

The greenhouse parameter acquisition module is used as the terminal module of the system. This design program needs to be downloaded to the $\mathrm{CC} 2530$ microcontroller for driving. Therefore, IAR software is required to compile the program to implement this function. The flow chart for the temperature acquisition program is shown in Figure 6. Because the read and write data are binary, only one bit can be read and written, for a total of 8 bits. Subsequently, the temperature, which is an ASCII value, is read converted to the sensor readout value. For testing convenience, the temperature value is sent to the computer through the serial port for a display to determine if there is a problem with the acquisition program. In the subsequent networking process, the collected data does not need to be sent to the serial port; instead, the data are sent to the sink node. The data are read and sent again after a delay, and the next data gain is performed to ensure the dynamic update of the data. The values measured by the program are accurate to one decimal place. The humidity and light data collections are similar to that for temperature.

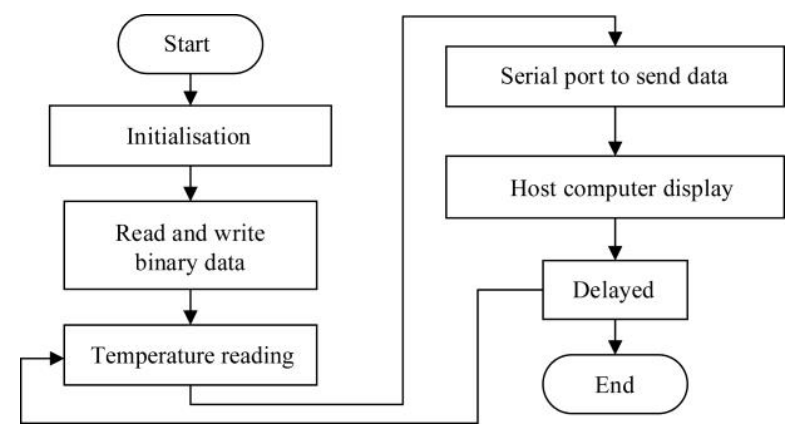

Figure 6 Temperature acquisition program flow chart

\subsection{Host computer monitoring software}

The host computer sends commands and acquires signals through the nodes of the routing station. Users can analyze the 
collected information link to the host computer ${ }^{[29]}$. The host computer has three functions. First, it displays the monitoring data in real-time. Second, three sets of parameters can be drawn as a line graph displayed in real-time, and the change of parameters can be conveniently observed. Finally, performance thresholds can be set.

The alarm operation is performed when the monitored data is not within the previously set range. Three windows are designed for this function, the monitoring data window, the line graph dynamic display window, and the threshold setting window and the alarm window. Three create three new form applications for the same project, Form 1, Form 2, and Form 3. Write three form application design features corresponding to the code compilation interface. The Form1 window is the display monitor data window. Drag three text box controls in the window to display three environment parameters. Then drag a button to control the opening and closing of the serial port. Clear the data in the three text boxes to conveniently display the collected data. Determine if the program is connected to the serial port.

If there is no connection, click "Start" to display "Error". If connected, the program can read the serial port number and accept the data transmitted by the serial port. After sorting the data, the programs are displayed in the three text boxes. Form 2 window is a dynamic display window for line drawings and is mainly used for timer controls and chart controls in VS. Chart controls are controls for drawing on the VS c\# environment. A chart control is dragged to the window to configure graphics, lines, and other related configurations and code. The timer control is a timer in c\#. Its main function is to execute the timer event written into the timer control at regular intervals. Form 3 window is a threshold setting and alarm window. The three sets of data received through the serial port under Form 1 window are called. The data in the text box is entered as the comparison range in this window interface. The timer is started, the data received by the serial port in the display window is called by the delegation and converted to integer or float type, and calculations performed with the input data. A comparison program is added to the timer event. If the value is in range, the timer will execute. An event is always calling and comparing the next set of data. If it is not within range, an alert will be created.

\subsection{Adaptive fuzzy-PID algorithm for data handling}

A strategy to further reduce data traffic and power consumption is the intelligent application method and sensor fusion shown ${ }^{[30,31]}$. PID controllers are the most widely used control loops because they are easy to design, simple in structure, and provide good control system performance. However, sometimes PID controllers cannot achieve a satisfactory control performance when the model is nonlinear and uncertain. Fuzzy control theory was first presented by Prof. Zadch, who developed a model that cannot be precisely described by mathematic ${ }^{[32,33]}$. This model can process characteristics using people's experiences to reflect and formulate certain control rules because PID controllers have a simple structure and the fuzzy method has a strong adaptability ${ }^{[34]}$.

In this model, the error and the error variation in the set value and the actual output value are used as the input of the fuzzy controller, and the variation in the PID parameter is used as the output of the fuzzy controller. The domain is divided according to different fuzzy states. For example, the domain is divided according to $\{N B, N M, \cdots, P M\}$. Each fuzzy state corresponds to a domain. When the input value of the input enters the fuzzy controller, the quantity is classified according to the different fuzzy state pairs to blur the exact value of the input quantity. The system error $E$ and error rate $E_{c}$ are defined as the domain of the fuzzy set. Its fuzzy subset is $E, E_{C} \in\{N B, N M, N S, Z O, P S, P M$, $P B\}$, and the subset elements represent negative, negative, negative, zero, positive, positive, and positive, respectively. Control rules are established for fuzzy PID controllers, as shown in Tables 1-3. The variation in the PID parameter is refined by using the centroid method, and the output of the entire fuzzy.

Table $1 K_{P}$ fuzzy control rule

\begin{tabular}{ccccccccc}
\hline \multirow{2}{*}{$K_{P}$} & \multicolumn{7}{c}{$E_{C}$} \\
\cline { 2 - 8 } & & $N B$ & $N M$ & $N S$ & $Z O$ & $P S$ & $P M$ & $P B$ \\
\hline \multirow{4}{*}{$E$} & $N B$ & $P B$ & $P B$ & $P M$ & $P M$ & $P S$ & $Z O$ & $Z O$ \\
& $N M$ & $P B$ & $P B$ & $P M$ & $P S$ & $P S$ & $Z O$ & $N S$ \\
& $N S$ & $P M$ & $P M$ & $P M$ & $P S$ & $Z O$ & $N S$ & $N S$ \\
& $Z O$ & $P M$ & $P M$ & $P S$ & $Z O$ & $N S$ & $N M$ & $N M$ \\
& $P S$ & $P S$ & $P S$ & $Z O$ & $N S$ & $N S$ & $N M$ & $N M$ \\
& $P M$ & $P S$ & $Z O$ & $N S$ & $N M$ & $N M$ & $N M$ & $N B$ \\
& $P B$ & $Z O$ & $Z O$ & $N M$ & $N M$ & $N M$ & $N B$ & $N B$ \\
\hline
\end{tabular}

Table $2 K_{i}$ fuzzy control rule

\begin{tabular}{|c|c|c|c|c|c|c|c|c|}
\hline & \multirow{2}{*}{$K_{i}$} & \multicolumn{7}{|c|}{$E_{C}$} \\
\hline & & $N B$ & $N M$ & $N S$ & $Z O$ & $P S$ & $P M$ & $P B$ \\
\hline \multirow{7}{*}{$E$} & $N B$ & $N B$ & $N B$ & $N M$ & $N M$ & $N S$ & $Z O$ & $\mathrm{ZO}$ \\
\hline & $N M$ & $N B$ & $N B$ & $N M$ & $N S$ & $N S$ & $Z O$ & $Z O$ \\
\hline & $N S$ & $N B$ & $N M$ & $N S$ & $N S$ & $Z O$ & $P S$ & $P S$ \\
\hline & $Z O$ & $N M$ & $N M$ & $N S$ & $Z O$ & $P S$ & $P M$ & $P M$ \\
\hline & $P S$ & $N M$ & $N S$ & $Z O$ & $P S$ & $P S$ & $P M$ & $P M$ \\
\hline & $P M$ & $Z O$ & $Z O$ & $P S$ & $P S$ & $P M$ & $P B$ & $P B$ \\
\hline & $P B$ & $Z O$ & $Z O$ & $P S$ & $P M$ & $P M$ & $P B$ & $P B$ \\
\hline
\end{tabular}

Table $3 K_{D}$ fuzzy control rule

\begin{tabular}{ccccccccc}
\hline \multirow{2}{*}{$K_{D}$} & \multicolumn{7}{c}{$E_{C}$} \\
\cline { 2 - 9 } & $N B$ & $N M$ & $N S$ & $Z O$ & $P S$ & $P M$ & $P B$ \\
\hline \multirow{4}{*}{$E$} & $N B$ & $P S$ & $N S$ & $N B$ & $N B$ & $N B$ & $N M$ & $P S$ \\
& $N M$ & $P S$ & $N S$ & $N B$ & $N M$ & $N M$ & $N S$ & $Z O$ \\
& $N S$ & $Z O$ & $N S$ & $N M$ & $N M$ & $N S$ & $N S$ & $Z O$ \\
& $Z O$ & $Z O$ & $N S$ & $N S$ & $N S$ & $N S$ & $N S$ & $Z O$ \\
& $P S$ & $Z O$ & $Z O$ & $Z O$ & $Z O$ & $Z O$ & $Z O$ & $Z O$ \\
& $P M$ & $P B$ & $N S$ & $P S$ & $P S$ & $P S$ & $P S$ & $P B$ \\
& $P B$ & $P B$ & $P M$ & $P M$ & $P M$ & $P S$ & $P S$ & $P B$ \\
\hline
\end{tabular}

The PID control system is calculated by the PID control algorithm to realize the control of the actuator. The domain of the errors $E_{C}$ and error $E$ as input is, the domain of the parameter $K_{p}$ as output is $\{-0.6,0.6\}$, the domain of the parameter $K_{i}$ is $\{-0.12$, $0.12\}$, and the domain of the parameter $K_{D}$ is $\{-6,6\}$. Fuzzy rules are added to the fuzzy controller through MATLAB's fuzzy logic toolbox. It can be seen that the conventional PID controller only takes about $300 \mathrm{~s}$ to reach the target temperature, while the fuzzy PID control system takes more than $500 \mathrm{~s}$. When the temperature reaches the target value, the conventional PID control system has a large overshoot and obvious ups and downs, which require a long settling time, while the temperature curve of the fuzzy PID control system is smoother and more stable, and the overshoot is reached after reaching the target temperature. Under the control of the fuzzy PID controller, the overshoot is small and the stability is good.

\subsection{Communication module design}

ZigBee technology is an important part of the Internet of 
Things. It has been widely used in industry, agriculture and other fields because of its advantages of low cost, low power and low latency ${ }^{[11,35]}$. ZigBee is a simple, low power consumption, and practical economical wireless communication technology, which is more suitable for the positioning of scenic spots in a specific outdoor area, with the advantages of strong self-organization and high scalability ${ }^{[36,37]}$. This proposed design uses Zigbee wireless transmission technology for greenhouse the environmental control of the greenhouse. The bottom layer of the ZigBee protocol architecture uses the IEEE 802.15.4 physical layer (PHY layer) and the medium layer (MAC layer). On this basis, the design establishes its network layer (NWK), which uses the layer framework to form the Zigbee protocol architecture. The wireless communication module designed is TI's Z-Stack design. The protocol stack is based on this design. APP is the application layer directory, and NWK is the network directory layer. The recording is primarily mainly developed in the APP layer compiler.

The wireless communication flow chart is shown in Figure 7. A well-integrated data takeover program is added in the application layer directory (APP) to complete the realize data collection of the terminal node. Carrying out the program is mainly performed in the main conducted directory. After the initialization is complete, the coordinator builds the network and the program assigns itself the unique network address of $0 \times 0000$ as the unique network address. The address is used to distinguish the Zigbee device, and the Zigbee device is divided into a MAC address and a website address.
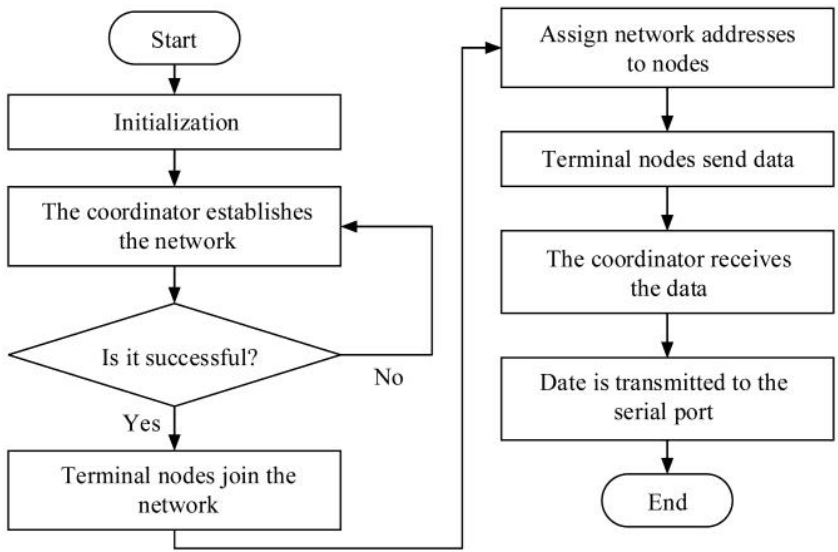

Figure 7 Wireless communication flow chart

The MAC address has 64 bits, and the website address has only 16 bits. Therefore, this program will generally include the URL. After the network is successfully connected, the terminal node or a router node can obtain a 16-bit web address, and the website program can complete the sending and receiving of data. After the network connection is successful, the terminal node sends data, and the coordinator receives the data and transmits it to the serial port.

\section{Test results of the greenhouse monitoring system}

The temperature sensor DS18B20, the humidity sensor DHT11, and the luminance sensor GY-302 are connected to the cc2530 as originally designed. The air temperature, humidity and light radiation sensors data are uploaded and saved every five minutes after the required experimental data are collected, which will eventually be available for indoor and outdoor air temperature and light intensity. The integrated $\mathrm{c}$ parameter acquisition program is downloaded to the microcontroller, and the microcontroller is connected to the computer through a USB cable to implement serial data transfer. After booting up, the serial debugging assistant is opened, and the "Open Serial Port" option is selected. A baud rate of 115200 was selected, and the situation was observed. The temperature, humidity, and illumination data are separated by commas. One group was collected every two seconds. To validate the designed data acquisition module, the system needs to be tested with two CC2530s, with one CC2530 serving as the coordination node to receive the data and upload it to the serial port. A terminal sensor was used for collecting environmental parameters. The designed wireless communication module program was downloaded to the coordinating node and the endpoint. The terminal node was placed remotely and the coordinator is connected to the computer.

The sensor is connected to the microcontroller. The integrated parameter acquisition program is downloaded to the CC2530. Next, the designed host computer program is opened, and the main interface data monitoring window appears. "Start" option is selected to display the monitoring results. Here, we can find data changes, real-time displays, and dynamic update monitoring data. The host computer software draws the collected data into a graphical dynamic display in real-time. It is updated every two seconds. The "Set Threshold" button in the monitor data display window is selected to open the threshold settings and alarm. The "Open" option is selected to open the alarm function and monitor the data in real-time. When the temperature data is higher than the input range, an alarm interface will pop up

\section{Conclusions}

The development of an adaptive microclimate system in the greenhouse is a crucial control issue for the successful growth of crops. This study proposed a wireless monitoring system for controlling greenhouse environmental conditions in order to monitor the microclimate condition, by analyzing the parameters, selecting various sensors and designing host computer monitoring. The test results showed that each sensor node operates normally and accurately collect multiple greenhouse parameters simultaneously. The aggregation node can receive data accurately and regularly. The host computer software can complete functions such as data display, waveform display, and threshold alarm. The proposed fuzzy-PID controller was easy to design and adaptive to measurement errors of the sensors. The proposed adaptive control system based on WSNs can calculate and preserve an optimal microclimate condition in the greenhouse.

\section{Acknowledgements}

This work is financially supported by the National Natural Science Foundation of China (No. 31901400), Zhejiang Province Natural Science Foundation (No. LQ20C130008), the Key Laboratory of Equipment and the Informatization in Environment Controlled Agriculture, Ministry of Agriculture and Rural Affairs (No. 2011NYZD1802).

\section{[References]}

[1] $\mathrm{Hu} \mathrm{H} \mathrm{G}, \mathrm{Xu} \mathrm{L} \mathrm{X,} \mathrm{Wei} \mathrm{R} \mathrm{H,} \mathrm{Zhu} \mathrm{B} \mathrm{K.} \mathrm{Multi-objective} \mathrm{contro}$ optimization for greenhouse environment using evolutionary algorithms. Sensors, 2011; 11(6): 5792-5807.

[2] Márquez Vera M A, Ramos-Fernández J C, Cerecero-Natale C F, Lafont F, Balmat J F, Esparza J I. Temperature control in a MISO greenhouse by inverting its fuzzy model. Computers and Electronics in Agriculture, 2016; 124: $168-174$.

[3] Li Z G, Andrews J, Wang Y Q. Mathematical modelling of mechanical damage to tomato fruits. Postharvest Biology and Technology, 2017; 
126: 50-56.

[4] Bai X Z, Liu L, Cao M Y, Panneerselvam J, Sun Q, Wang H X. Collaborative actuation of wireless sensor and actuator networks for the agriculture industry. IEEE Access, 2017; 5: 13286-13296.

[5] Lopes Toledo A, Lèbre La Rovere E. Urban mobility and greenhouse gas emissions: status, public policies, and scenarios in a developing economy city, Natal, Brazil. Sustainability. 2018; 10(11): 1-24.

[6] Yin J, Yang Y, Cao H, Zhang Z. Greenhouse environmental monitoring and closed-loop control with crop growth model based on wireless sensors network. Transactions of the Institute of Measurement \& Control, 2015; 37(1): 50-62

[7] Ding Y, Wang L, Li Y W, Li D L. Model predictive control and its application in agriculture: A review. Computers and Electronics in Agriculture, 2018; 151: 104-117.

[8] Wang L N, Zhang H H. An adaptive fuzzy hierarchical control for maintaining solar greenhouse temperature. Computers and Electronics in Agriculture, 2018; 155: 251-256.

[9] Ferentinos K P, Katsoulas N, Tzounis A, Bartzanas T, Kittas C. Wireless sensor networks for greenhouse climate and plant condition assessment. Biosystems Engineering, 2017; 153: 70-81

[10] Gao P Q, Tian Z D, Gao X W. Network teleoperation robot system control based on fuzzy sliding mode. Journal of Advanced Computational Intelligence \& Intelligent Informatics, 2016; 20(5): 828-835.

[11] Tang Y L, Lian H H, Li L X, Wang X J, Yan X X. A randomness detection method of ZigBee protocol in a wireless sensor network. Sensors, 2018; 18(11): 3962

[12] Qi W H, Zong G D, Karimi H R. L $\infty$ control for positive delay systems with semi-Markov process and application to a communication network model. IEEE Transactions on Industrial Electronics, 2019; 66(3): 2081-2091.

[13] Alfonso G B, Pablo C, Hyun Y, Juan C. GreenVMAS: Virtual organization based platform for heating greenhouses using waste energy from power plants. Sensors, 2018; 18(3): 861-881.

[14] Wang L, Zhang $H$. Sliding mode control with adaptive fuzzy compensation for uncertain nonlinear system. Mathematical Problems in Engineering, 2018; (PT.17): 2342391.1-2342391.6.

[15] Qi W H, Park J H, Zong G D, Cao J D, Cheng J. Anti-windup design for saturated semi-Markovian switching systems with stochastic disturbance. IEEE Transactions on Circuits and Systems II: Express Briefs, 2019; 66(7): 1187-1191.

[16] Tian Z D, Li S J, Wang Y H, Sha Y. A prediction method based on wavelet transform and multiple models fusion for chaotic time series. Chaos Solitons \& Fractals, 2017; 98: 158-172.

[17] Kamilaris A, Prenafeta-Boldú F X. Deep learning in agriculture: A survey. Computers and Electronics in Agriculture, 2018; 147: 70-90.

[18] Anna C, Salah S, Brett W. Machine learning approaches for crop yield prediction and nitrogen status estimation in precision agriculture: A review. Computers and Electronics in Agriculture, 2018; 151: 61-69.

[19] Manonmani A, Thyagarajan T, Elango M, Sutha S. Modelling and control of greenhouse system using neural networks. Transactions of the Institute of Measurement and Control, 2016; 40(3): 918-929.

[20] Nachidi M, Rodriguez F, Tadeo F, Guzman J L. Takagi-Sugeno control of nocturnal temperature in greenhouses using air heating. ISA
Transactions, $2011 ; 50(2): 315-320$

[21] Zheng X L, Wang X Y, Yin Y F, Hu L L. Stability analysis and constrained fuzzy tracking control of positive nonlinear systems. Nonlinear Dynamics, 2015; 83(4): 22509-22522.

[22] Ramírez-Arias A, Rodríguez F, Guzmán J L, Berenguel M Multiobjective hierarchical control architecture for greenhouse crop growth Automatica, 2012; 48(3): 490-498.

[23] Yau H T, Chen C L. Fuzzy sliding mode controller design for maximum power point tracking control of a solar energy system. Transactions Institute of Measurement \& Control, 2012; 34(5): 557-565.

[24] Li X H, Cheng X, Yan K, Gong P. A monitoring system for vegetable greenhouses based on a wireless sensor network. Sensors, 2010; 10(10): 8963-8980.

[25] Tang Y, Ma X, Li M, Wang Y. The effect of temperature and light on strawberry production in a solar greenhouse. Sol. Energy, 2020; 195: 318-328.

[26] Ioslovich I, Gutman P O, Linker R. Hamilton-Jacobi-Bellman formalism for optimal climate control of greenhouse crop. Automatica, 2009; 45(5): 1227-1231.

[27] Ahmed H A, Tong Y X, Yang Q C, Al-Faraj A A, Abdel-Ghany A M Spatial distribution of air temperature and relative humidity in the greenhouse as affected by external shading in arid climates. Journal of Integrative Agriculture, 2019; 18(12): 2869-2882.

[28] Zhu X N, Ding B J, Li W J, Gu L Z, Yang Y X. On development of security monitoring system via wireless sensing network. J Wireless Com Network, 2018: 221.

[29] Bahloul M, Chrifi-Alaoui L, Drid S, Souissi M, Chaabane M. Robust sensorless vector control of an induction machine using multiobjective adaptive fuzzy luenberger observer. ISA Transactions, 2018; 74: 144-154.

[30] $\mathrm{Su} \mathrm{Y,} \mathrm{Xu} \mathrm{L,} \mathrm{Li} \mathrm{D.} \mathrm{Adaptive} \mathrm{fuzzy} \mathrm{control} \mathrm{of} \mathrm{a} \mathrm{class} \mathrm{of} \mathrm{MIMO} \mathrm{nonlinear}$ system with actuator saturation for greenhouse climate control problem, IEEE T. Autom. Science Eng. 2016; 13(2): 772-788.

[31] Kudinov Y I, Kolesnikov V A, Pashchenko F F, Pashchenko A F, Papic L. Optimization of fuzzy PID controller's parameters. Procedia Computer Science, 2017; 103: 618-622.

[32] Wang Y Z, Jin Q B, Zhang R D. Improved fuzzy PID controller design using predictive functional control structure. ISA Transactions, 2017; 71(Part 2): 354-363.

[33] Precup R E, Hellendoorn H. A survey on industrial applications of fuzzy control. Computers in Industry, 2011; 62(3): 213-226.

[34] Akram U, Khalid M, Shafiq S. An improved optimal sizing methodology for future autonomous residential smart power systems. IEEE Access, 2018; 6: 5986-6000.

[35] Ma X P, Dong H H, Tang J Q, Jia L M, Qin Y, Cheng R J. Two-Layer hierarchy optimization model for communication protocol in railway wireless monitoring networks. Wireless Communications \& Mobile Computing, 2018; 2018: 9516342.

[36] Liu F C, Liu Y L, Jin D H, Jia X Y, Wang T T. Research on workshop-based positioning technology based on internet of things in big data background. Complexity, 2018; 6: 1-11.

[37] Meka S, Fonseca, Jr. B. Improving route selections in ZigBee wireless sensor networks. Sensors, 2020; 20(1): 164. 